\title{
The developing pattern of clinical pathology
}

From time to time we are given an opportunity of commenting upon current developments of great interest to clinical pathologists, and in this issue we are happy to print on page 3 extracts from the Presidential Address to the sixth meeting of the International Society of Clinical Pathologists recently held in Rome. Professor Welsch has sketched the future role of the clinical pathologist and we are taking this opportunity to express some of the ideas which we see emerging in the highly developed countries. The situation in the underdeveloped countries and those parts of Europe and North America where the pattern of populations and great distances make the picture quite different may require a quite different approach.

The identification of the origins of ill health, the way in which extrinsic and intrinsic factors distort the physical and mental patterns of the normal person are the business of the clinical pathologist. Scientific developments over the past decades have not altered that business, they have only refined and multiplied the procedures by which the end is achieved.

The enormous expansion of the part that the laboratory plays in diagnosis and control of therapy has lead arbitrarily to the creation of specialities within the total specialty of clinical pathology. Custom and practical experience have resulted in the development of four main fields of specialization; histopathology, microbiology, haematology, and chemical pathology. It is customary now for a pathologist to concentrate on one of these areas though he may well, and, indeed should have, experience in others.

Anyone with more than superficial interest in any one of the fields knows how tenuous the divisions are and so it remains vital that we should be able to talk each other's 'language'. The application of basic science to biological problems has enormously enhanced the part that biochemically and biophysically trained members of the consortium play in the day-to-day work in the laboratory.

The tools of the trade have increased in complexity and the proper mastery of these tools increasingly absorbs time. There is a feeling that the handling of the tools can be left wholly to the technician, but interpretation, the end point of our work, cannot be divorced from an understanding of procedure. There is a dangerous conceit that certain types of clinical pathological investigation may profitably be divorced from a big, well-equipped laboratory and attached to and controlled by a small specialized clinical department. The most cursory examination will show that this is uneconomic in capital cost, in space, and in the effective use of skilled technical assistance, always in short supply. Even worse, method deteriorates and technicians lose their fire. We must be alert, however, to the possibilities that the scientific yearnings of our clinical colleagues do not spring from our own inadequacy in keeping the quality of both our advice and service abreast of the rapid moves of science.

Perhaps the design of the laboratory of the future and of clinical pathology as a subject have to be examined from other points of view too. Patients are being investigated in three quite separate ways. First is the method by which the patient is investigated by a clinician, and a multiplicity of unconnected tests is requested. The patient may have various blood examinations e.g., blood count; then a blood urea in the biochemistry department; a nasal swab and vaginal specimen for cytology may be taken in another department; and perhaps a biopsy or scraping using a third department. All the relevant data are collected and assessed by the clinician. Secondly, a patient may be investigated by the members of a specialized department interested in only one system and its functioning, for example, the genito-urinary tract, so that renal function tests, bladder tests, and so on, including all the relevant biochemical and other investigations, will all be done by one team. Another team may be investigating, again using multidiscipline techniques, the patient's respiratory or cardiovascular function. Furthermore, a third team may be investigating his gastrointestinal tract involving such widely different techniques as monitoring stomach function, taking gut biopsies, examining pan- 
creatic secretions, faeces, and liver function. In this way a pathology department may become a department of clinical investigation or a department of laboratory medicine to investigate the normal and abnormal physiology or pathology of a given system. This may well be a rapidly developing pattern. Thirdly, we are about to undertake screening techniques on the lines already adopted by some American hospitals in which any patient who is seen by a general practitioner or a hospital doctor will be screened for a large number of parameters, whichever are, in fact, available. The development of automation in the laboratory has made it possible to screen a patient for anything from six to 20 biochemical analyses which might rule out or perhaps highlight a particular disorder. Of course, screening procedures have always been part of a hospital service in as much as taking the temperature and blood pressure and simple estimations of haemoglobin are all routine screening processes.

The development of pathology services will, therefore, follow a combination of these patterns and some visualize that within the next 10 years laboratories will have to be organized as 'cold', routine laboratories where straightforward, routine tests in which a few major investigations, such as of haemoglobin, blood urea, blood sugar, are carried out on the majority of patients. Overlapping technical interests will be catered for by joint departments. The major part of the acute or 'hot' work of the hospital will be concentrated on the intensive care unit where a special laboratory will cover all the urgent investigations which might be wanted at a moment's notice.

We believe that the future pathology services must, therefore, be conceived as a major unit easily accessible to the whole of the hospital staff, to outside general practitioners, medical officers of health, industrial medical officers, and organized on such a basis that there is a quick turnover of the simple, routine testing with sufficiently highly qualified medical and technical staff to be able to investigate fully all the abnormalities associated with the four major disciplines as they are now organized. One could easily in this way visualize large-scale screening of populations as part of the 'cold' laboratory's work, and this would include such things as population surveys for diabetes; and vaginal cytology, now such a political issue, would become part of the screening facilities of the histopathological department and/or the biochemical department if enzyme screening were to become more effective.

The staff of the laboratory of any large hospital of over 800 beds cannot, therefore, be less than four senior medical consultant pathologists each covering a major discipline but also well acquainted with the particular techniques of the other disciplines. There should be a senior non-medical biochemist who would work with the medical chemical pathologist, a senior biophysicist for the isotope and similar techniques, an adequate number of pathologists in training, and a full quota of trained scientific assistants or senior technicians together with junior technicians in training to cover the work of the department. It is essential that the department, particularly where the acute work is concentrated, must be active over the 24 hours of the day although a full service can only be supplied during extended ordinary working hours for the cold routine work. It may be that in order to cover the increasing demands, an automated laboratory would be required and would have to work on a shift system covering the hours of say 8 a.m. to 10 p.m. with a fuller staff than is now available for evening work. By this means automation could be used to its fullest extent and enable the staff to process many more tests than can be done by manual methods and with full quality control.

The International Society of Clinical Pathology at its last meeting in Rome set up an International Standards Committee which, it is hoped, will unify the work of so many other international standard committees and this will undoubtedly help in the standardization and upgrading of the work of laboratories all over the world. These are exciting times and we, the clinical pathologists, must grasp the opportunities they offer. 